\section{Probes in Huntington's chorea}

SIR-Over two years ago Nature published the first linkage of a marker with Huntington's chorea and underlined its importance and significance with a full and lucid annotation.

Under the expectation that the probe presented would be of some clinical use under certain conditions and that it would be followed by closer probes, some of which would bracket this locus, we organized a register of cases in this Region (population 2,500,000) and acquired funds and limited facilities allowing us to start to apply DNA techniques to the practical problems presented by patients and their relatives. We now know of over 300 cases in 140 families, and laboratory work started in October. We are not inexperienced and many members of the families we are trying to help are responsible and intelligent.

On asking for the probe we received a letter from Dr Gusella stating that there were ethical problems in its use, that further research on heterogeneity was needed and that we could only have it if we did not use it clinically. We are of course aware that on its own, it is too far from the locus to be of clinical value in most families. We are also aware that cases are often misdiagnosed; and that this is sometimes euphemistically included under heterogeneity.

No novel ethical problems are involved in initiating work by the cautious application of new and unreliable techniques. Any relevant test on a patient when the diagnosis is in doubt must be followed by news which is either good or bad. Suicide in response to bad news is a rational anxiety but rare in medical practice. If Wasserman had published his test for syphilis, which was far from reliable, in a way which delayed its application, neurosyphilis might now be more common than Huntington's chorea. This infectious disease involved far more difficult problems in handling patients and their families; many individuals must have been distressed by investigations based on error and there were probably a few suicides. But the disease is now rare in Northern Europe. In no field of effective medicine can techniqes be applied without casualties.

Quite apart from these serious ethical issues now arising in the field of DNA, Nature is a scientific journal and it should be assumed that others will be able to confirm the claims made in articles it publishes. The time involved in preparing a paper of sufficient standard is so long that there is little justification for withholding information or resources. It would hardly be considered ethical to publish an article on an interesting star, but to withold its position to allow a monopoly of further research or development. In this new field Cell has already given a lead - perhaps Nature could follow and neither publish nor refer to work which cannot be validated.

D.C. WATT R.H. LINDENBAUM J.A. JONASSON J.H. EDWARDS

Medical Genetics Unit,

Churchill Hospital,

Headington,

Oxford $O X 3$ 7LE, UK

GuSElla RePlies-Watt et al. appear to confuse scientific research with clinical practice. Our report in Nature did not present a presymptomatic diagnostic test for Huntington's disease (HD), but demonstrated linkage of HD to a genetic marker defined by the DNA probe G8 on chromosome $4^{\prime}$. We agree wholeheartedly that if scientific reports are published it should be possible to repeat and confirm them. To this end, we have distributed the G8 probe to over 30 laboratories in 10 countries. We are also collaborating with many other investigators to apply the probe to additional HD families. In all cases, with the agreement of the investigators involved, the work is being performed for research purposes only, not to derive predictive information for delivery to "at risk" individuals.

Our reasons for limiting clinical use of the marker were referred to in the original report of its discovery in Nature. There is considerable precedent in genetics, including human genetic disease, for nonallelic heterogeneity in which mutations at two or more separate loci result in the same phenotype. If a second unlinked locus were the cause of HD in some families, predictive testing for these using the G8 marker would be no better than guesswork, and many "at risk" family members might become unnecessary "casualties" to false information.

Heterogeneity can only be ruled out by typing individuals of defined HD status in fairly large pedigrees, unlike those described to us by Dr Edwards and his colleagues. When we received the request from this group to proceed with predictive testing, linkage of the marker to the disease locus had only been published for four independent families, far too few to conclude that a second HD locus does not exist in a significant proportion of families $^{1.2}$. Efforts to test the possiblity of heterogeneity are ongoing in many centres and should provide conclusive results in the near future. We have not performed any presymptomatic or prenatal diagnoses using the marker and feel that universal clinical application of the G8 probe in HD remains premature.

Unlike Watt et al., we feel that there are significant considerations in the presymptomatic diagnosis of HD that make it similar to several other neurological and behavioural disorders for which markers may soon be found, but that distinguish it from a communicable disease like syphilis. $\mathrm{HD}$ is a late onset condition that affects the victim both physically and mentally, but can only transmitted to progeny. In addition to motor disturbance, psychiatric symptoms are common, and the risk of suicide is significantly increased ${ }^{3.4}$. As there is no effective therapy for the disorder, any presymptomatic testing is purely informational and will not affect treatment. We believe that when accurate presymptomatic diagnosis is possible, it should be made available for the benefit of those "at risk" individuals who request it, rather than for the purposes of physicians, other family members, insurance companies, employers, or governments. A positive test would indicate to an apparently healthy individual that he or she has a very high probability of becoming progressively disabled, both mentally and physically, later in adult life, and that any children might await a similar fate. In doing what is best for the patient, it is the obligation of the healthcare professional to deliver this disruptive and potentially devastating information in a manner which minimizes its many possible detrimental effects on both the individual and his or her family.

When accurate presymptomatic testing is possible, the appropriate setting will be one which includes adequate provisions for pre- and post-test counselling as well as long-term psychological support ${ }^{5}$. We have been working closely with the HD voluntary organizations who represent patients and their families to ensure that the needs of the "at risk" individuals will be met when presymptomatic testing can be initiated. We have formally raised these issues for consideration at international meetings of the World Federation of Neurology Research Group on Huntington's Disease and of the International Huntington's Association and have also privately solicited input from many expert neurologists, psychiatrists and geneticists.

It is our intention that when accurate presymptomatic testing is possible, the G8 probe will be made available to any qualified centre through the Massachusetts General Hospital Committee for Presymptomatic HD Testing. In the meantime, we regret having to place any restrictions on the use of the G8 probe, but these are not ones which should delay scientific progress or test development. We and others are actively searching for additional markers that will increase both the accuracy and applicability of the potential presymptomatic test, but the heterogeneity issue must be resolved before proceeding to preclinical diagnosis. We feel a mor- 